\title{
Synthetic approaches to dexmedetomidine (review)
}

\author{
Mária Bobošíková, František Mathia, Daniel Végh, \\ Štefan Marchalín, Michaela Halinkovičová \\ Institute of Organic Chemistry, Catalysis and Petrochemistry, Faculty of Chemical and Food \\ Technology, Slovak University of Technology, Radlinského 9, SK-812 37 Bratislava, Slovak Republic \\ stefan.marchalin@stuba.sk
}

\begin{abstract}
The review is an attempt at critical evaluation of known synthetic procedures used for laboratory and industrial preparation of a synthetic antihypertensive - Dexmedetomidine as to their complexity, published yield and atom economy.
\end{abstract}

Keywords: Dexmedetomidine, medetomidine, synthesis, sedatives

\section{Introduction}

Dexmedetomidine (1) - systemic name $(S)-4-[1-(2,3-$ dimethylphenyl)ethyl]-3 $H$-imidazole - represents a highly potent " $\alpha_{2}$-adrenoreceptor agonist", used in general management of patients undergoing surgery, as well as in intensive medicine. (Cormack et al., 2005) Dexmedetomidine has been commercially available under the trade names Precedex or Dexdor; inlike similar " $\alpha_{2}$-adrenoreceptor agonists" it offers sufficient sedation accompanied by relatively infrequent side effect - "respiratory depression", allowing surgeries without artificial ventilation of the patient. Fig. 1

\section{Synthetic approaches to Dexmedetomidine}

Dexmedetomidine (1) was first prepared in 1987 by resolution of racemic medetomidine by enantiomerically pure $(+)$-tartaric acid. The thus obtained diastereomeric salts were separated by fractional crystallization in ethanol. (GB2206880)

Preparation of Dexmedetomidine (1) published so far are based on fractional crystallization of diastereomeric salts of medetomidine (2) with the some optically active acids. An asymmetric synthesis of an optically pure product is yet to be published. That is why our analysis concentrates first on synthetic procedures leading to racemic medetomidine (2) and finally on methods of separation of its enantiomers.

The medetomidine (2) was first prepared in the course of a diversified search for novel, imidazole based, antihypertensives. (US4544664) (Scheme 1) In its first steps the procedure involves a twofold addition of Grignard reagents, first that of the substituted arylmagnesium bromide (4) to the methyl ester of the 4-imidazolecarboxylic acid (3), followed by that of methylmagnesium bromide to the formed ketone (5), resulting in the formation of the tertiary alcohol (6). Alcohol (6) has to be separated from the reaction mixture by a column chromatography, to the detriment of laboratory and even more of industrial manufacturing process. After dehydration of alcohol (6), followed by reduction of the ensuing double bond of the substituted styrene ( 7 ) medetomidine (2) is obtained. Details of hydroxylic group elimination or double bond reduction have been forgone by the authors. They give neither yields of single steps, nor the overall yield, making their procedure rather less attractive. The procedure nevertheless remains historically the first one published.

Kudzma and coworkers in 1991 selected another approach to the tertiary alcohol (6). (Kudzma et al., 1991) The appropriately protected imidazole (9) was used as nucleophile, whereas the chloride of benzoic acid (10) served as an electrophile. Protecting

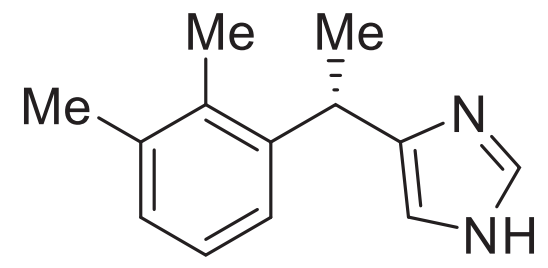

1

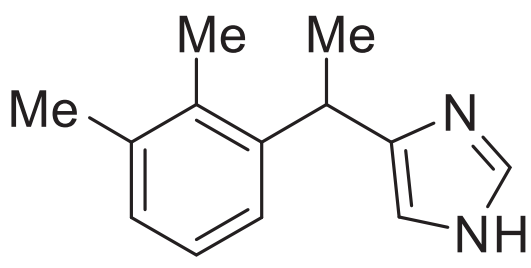

2

Fig. 1. Dexmedetomidine (1) and Medetomidine (2). 
<smiles>Cc1cccc(C(C)(O)c2c(C)cccc2C(O)(O)c2c[nH]cn2)c1C</smiles>

(3)

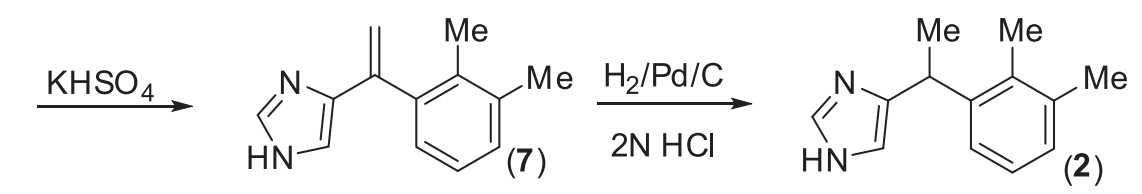

Scheme 1<smiles>CO[S+]([O-])(OC)n1ccnc1</smiles>

(8)

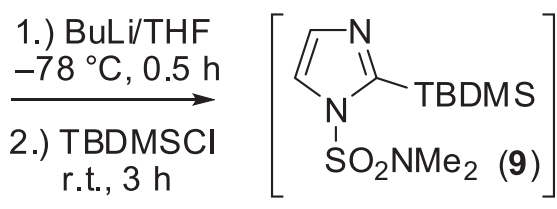
r.t., $3 \mathrm{~h}$

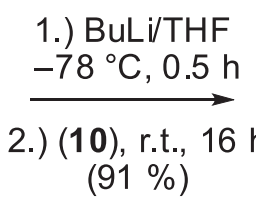<smiles>COS(=O)(=O)n1cc(C(=O)c2cccc(C)c2C)nc1SC(C)(C)C</smiles>

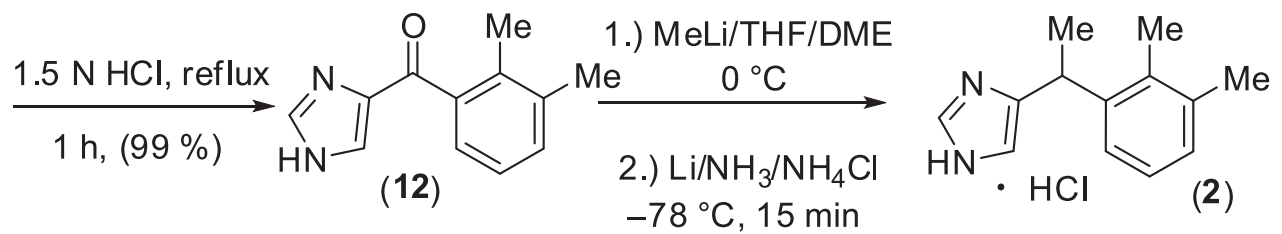<smiles>Cc1cccc(C(=O)Cl)c1C</smiles>

3.) ${ }^{\mathrm{i}} \mathrm{PrOH} / \mathrm{Et}_{2} \mathrm{O} / \mathrm{HCl}$

$(88 \%)$

Scheme 2<smiles>Cc1cccc(N(Br)CBr)c1C</smiles>

1.) THF, $0^{\circ} \mathrm{C},(52 \%)$

2.) $\mathrm{MnO}_{2}$, dioxane, rflx $(90 \%)$<smiles>CCn1cnc(C(=O)c2cccc(C)c2C)c1</smiles>

(14)

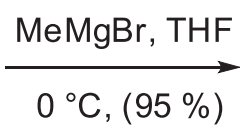

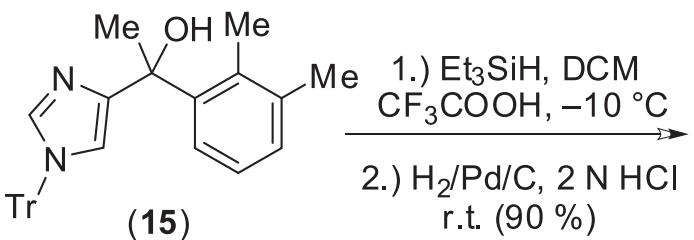<smiles>Cc1cccc(C(C)c2c[nH]cn2)c1C</smiles>

Scheme 3

groups at the imidazole skeleton of the ketone (11) were removed by the action of $1.5 \mathrm{~N}$ hydrochloric acid The thus obtained deprotected ketone (12) was then transformed to the desired hydrochloride of medetonidine (2) by a sequence of reactions. This approach allowed a very good, $79 \%$ overall yield of the target compound, although it involved at least three synthetic steps, all highly sensitive to the presence of moisture and carried out at low temperatures. Moreover, purification of ketone (11) too required the use of chromatography. (Scheme 2)
Cordi and coworkers too applied 2,3-dimethylphenylmagnesium bromide (4) as a nucleophile. (Cordi et al., 1996) (Scheme 3) The addition at the protected imidazolecarbaldehyde (13) proceeds however only in rather unsatisfactory yield of $52 \%$. In the next step a relatively cheap oxidant (pyrolusite) is used to convert the prepared secondary alcohol to the ketone (14), needed in the subsequent addition of methylmagnesium bromide. The thus prepared tertiary alcohol (15), after elimination of water and reduction of the 
double bond affords medetomidine (2) in the overall yield of $41 \%$. The biggest single drawback of this procedure is the need of protecting group at the nitrogen of imidazolecarbaldehyde (13) and poor yield of the addition of 2,3-dimethylphenylmagnesium bromide (4).

The same trityl protecting group at imidazole nitrogen was use also by the authors of the 2008 patent. (EP1918282) The imidazole ring enters the molecule of medetomidine (2) via the attack of nucleophile (16) at the electrophilic carboxylic functional group of the substituted benzaldehyde (17). (Scheme 4) The intermediary secondary alcohol (18) undergoes the same oxidation as in the previous example. After addition of methylmagnesium bromide the tertiary alcohol (15) is obtained, to be transformed by the standard elimination-reduction sequence to the desired product - medetomidine (2), in the $58 \%$ overall yield. In spite of the use two moisture-sensitive Grignard additions and atom economy lowered by the use of protecting group, the procedure offers a quite good overall yield. Another asset is the detailed and accurate description of every single synthetic step.
A patent from 2009 presents an alternative introduction of imidazole ring into the structure of product. (WO2009053709) (Scheme 5) The synthetic sequence starts by preparation of 2,3-dimethylbenzenecarbaldehyde (20) from 2,3-dimethylbenzyl bromide (19). The subsequent addition of methylmagnesium bromide to aldehyde (20) affords the secondary alcohol (21). The synthesis continues by substitution of the hydroxyl group by protected imidazole (22) in the presence of Lewis acid. The procedure is hampered by the use of moisture sensitive reagents in each step, use of molar equivalent of Lewis acid as well as by the need to protect the imidazole nitrogen. The published data also suffer from lack of yield data and sketchy description of experiments. On the other hand the procedure is quite fast (3 steps), proceeds at near laboratory temperature and gets on without a reduction step. Authors of the most recent preparation of medetomidine (2) elaborated a 9-step synthesis starting from the 2,3-dimethylbenzoic acid (23). (WO2011070069) (Scheme 6) The transformation of carboxylic acid (23) gives rise to activated derivatives (24) and (12), the latter serving for the preparation of nitroketone (25). Nitroketone is then utilized for the de novo

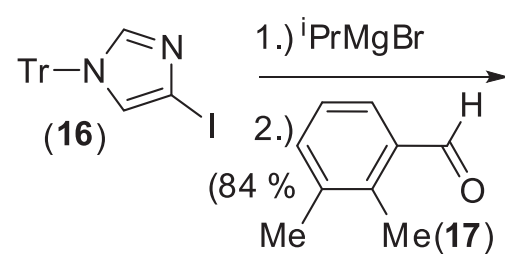<smiles>[3H]n1cnc(C(O)c2cccc(OC)c2C)c1</smiles>

(18)<smiles>Cc1cccc(C(=O)c2cn(C)cn2)c1C</smiles>

(14)

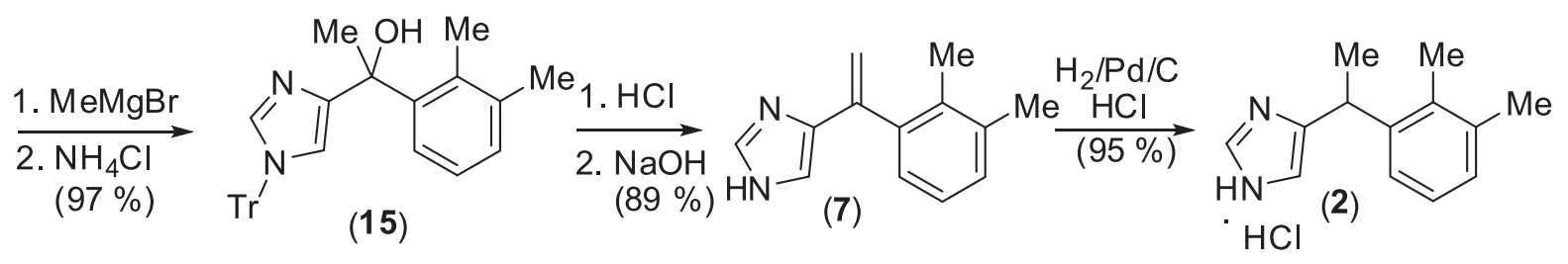

Scheme 4<smiles>CCON(O)CCO[N+](=O)[O-]</smiles><smiles>Cc1cccc(C=O)c1C</smiles><smiles>C[R18](Br)(Br)Cc1ccccc1</smiles><smiles>Cc1cccc(C(C)O)c1C</smiles>

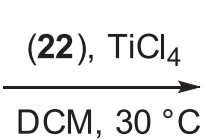<smiles>Cc1cccc(C(C)c2c[nH]cn2)c1C</smiles><smiles>[As]n1ccnc1</smiles>

(22)

Scheme 5 
construction of the imidazole ring, present in drug molecule (2). The second nitrogen of the imidazole ring comes from benzylamine. The reaction of benzylamine with nitroketone (25) affords the nitroenone (26), which after reduction with Raney nickel gives the corresponding endiamine (27). Cyclization of diamine (27) with ethyl orthoformate gives rise to the derivative (28), containing an imidazole ring. Next, a standard reaction sequence (addition-elimination-reduction) was used to prepare medetomidine (2) via the styrene derivative (29), in the $13 \%$ overall yield. Unfortunately the synthetic sequence is rather lengthy, affords low overall yield and requires two catalytic reductions. To make things worse the sequence does not allow the benefit of simple preparation of analogs, containing a substituted imidazole or benzene ring. It is thus difficult to see its advantages for either laboratory synthesis, or, due to its drawn-out and cost-intensive character, for industrial scale manufacturing.

\section{Separation of enantiomers of medetomidine}

Authors of the above mentioned patents from 1987 (GB2206880, US4910214) deployed for the separation of enantiomers of medetomidine (2) by (+)-tartaric acid recurring fractional crystallization of diastereomeric salts from the mixture of methanol and ethanol. By repeated crystallization of the salt formed from mixing equivalents of medetomidine (2) and tartaric acid authors succeeded in isolating Dexmedetomidine (1) in $17 \%$ overall yield. Although the number of successive crystallizations have not been specified, they stop when the value of product optical rotation no longer rises.

Another preparation involved the salts of medetomidine (2) with (+)-tartaric acid, (-)-mandelic acid and (-)-camphor-10-sulfonic acid. The best separation was achieved with tartrates. (US4910214) Comparison of relative bioactivity of enantiomers (after their liberation as free bases from diastereometic

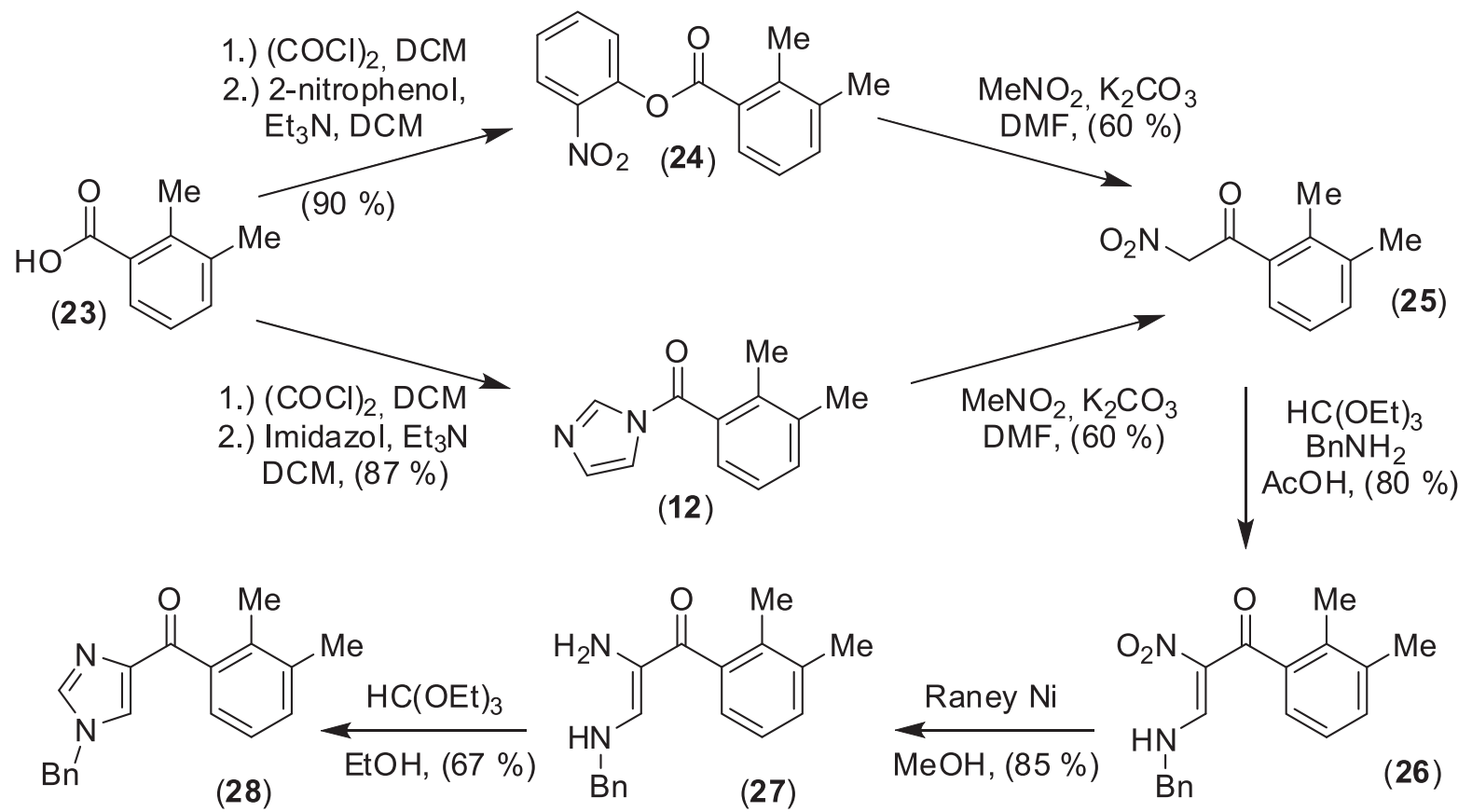<smiles>C=C(c1cn(Cc2ccccc2)cn1)c1cccc(C)c1C(=O)C(C)C(C)(C)Br</smiles>

Scheme 6 
salts) showed that $(S)$-enantiomer demonstrated by $50 \%$ higher in vitro activity than $(R)$-enantiomer, thus directing the interest of pharmaceutical companies towards the more active $(S)$-enantiomer of Dexmedetomidine (1).

However, the patent does not disclose the yield and enantiomeric purity of Dexmedetomidine.

Cordi and coworkers disclose preparation of Dexmedetomidine where resolution of medetomidine (2) was carried out by using mere 0.5 equivalent of (+)-tartaric acid in absolute ethanol to one equivalent of medetomidine (2). (Cordi et al., 1996) Dexmedetomidine $(+)$-tartarate was prepared after 4 crystallizations from ethanol in higher than $99 \%$ enantiomeric excess and $21 \%$ overall yield. Authors also describe the possibility to further improve the yield while preserving purity by markedly longer equilibration time of the mixture. As the process requires 5 days to provide Dexmedetomidine in very low overall yield it is industrially uneconomical.

The chinese patent also discloses preparation of Dexmedetomidine (1) where medetomidine (2) was resolved by using $S$-(+)-phosphoric acid-hydro-1,1' di-2,2'naphtyl ester $[S$-(+)-BNP] , D-(+)-dibenzoyl tartarate $[D-(+)$-DBTA $], L-(-)$-dibenzoyl tartarate [L-(-)-DBTA] or $R$-(-)-phosphoric acid-hydro-1,1' di-2,2'-naphthyl ester [R-(-)BNP]. (CN101671305) This process is also time consuming and requires highly expensive reagents thus making the process industrially uneconomical.

Needless to say it is advantagenous to develop a short, high yielding and industrially viable process for preparation of highly pure Dexmedetomidine which eliminates use of expensive reagents. It is therefore an object of the new invention to overcome or ameliorate at least one disadvantage of the prior art or to provide an useful alternative. Another object of the invention is to provide a novel, concise, high yielding commertially viable and industrially applicable process for resolution of medetomidine to provide Dexmedetomidine in high yield and with high enantiomeric excess. Yet another object of the present invention is to provide a process for preparation of Dexmedetomidine having desirable pharmacological activity and broad safety margins, without toxicity or unfavorable side effects.

In accordance with the above objectives, the newest patent provides a process for preparation of Dexmedetomidine. (WO2013069025) There are described five steps:

(i) reacting medetomidine with $D$-(-)-tartaric acid in the presence of solvent (the most preferred solvent is ethanol)

(ii) filtering the resulting solution (iii) neutralizing the filtrate with a base (preferred is inorganic base selected from alkali metal carbonates or alkali metal bicarbonates, e.g. sodium bicarbonate)

(iv) reacting the resulting product with $L$-(+)tartaric acid to provide dexmedetomidine $L-(+)$-tartarate

(v) reacting dexmedetomidine $L-(+)$-tartarate with a base (the most preferred base is sodium bicarbonate)

The process of the invention provides Dexmedetomidine in $47 \%$ yield and with enantiomeric excess of at least $99 \%$. The yield of Dexmedetomidine obtained by using the novel process is 2.2 times higher than that obtained by using resolution methods reported previously. All the steps of the process can be carried out in a single solvent and in a single base which are inexpensive. The process is thus economical. Dexmedetomidine is further converted into its hydrochloride salt. The pure Dexmedetomidine hydrochloride obtained by the above mentioned process may be formulated into a dosage form.

In spite of low yields of Dexmedetomidine (1) in in procedures involving separation of medetomidine enantiomers (2) no synthetic procedure is known, leading directly to the desired enantiomer, starting either from optically pure starting compound, or using chiral auxiliaries in e.g. double bond reduction.

\section{Acknowledgement}

This contribution is the result of the project: Research Center for Industrial Synthesis of Drugs, ITMS 26240220061, supported by the Research E Development Operational Programme funded by the ERDF. This work was also supported by the grant agency of Slovak Republic (Grant 1/0429/11) and by the Slovak research and development agency under the contract APVV-0204-10.

\section{References}

CN101671305

Cordi AA, Persignand T, Lecouvé JP (1996) Synth. Commun. 26: 1585-1593.

Cormack JR, Orme RM, Costello TG (2005) J. Clin. Neuro. 12: 375-378.

EP1918282

GB2206880

Kudzma LV, Turnbull Jr SP (1991) Synhesis: 1021-1022.

US4544664

US4910214

WO2009053709

WO2011070069

WO2013069025 